\title{
Brainstem Auditory Impairment in Very Preterm Infants who Experience Chronic Sublethal Hypoxia
}

\author{
James Ken Jiang ${ }^{1}$, Cui Wong ${ }^{2}$ and Ze Dong Jiang2* \\ ${ }^{1}$ School of Physiology, Pharmacology and Neuroscience, University of Bristol, UK \\ ${ }^{2}$ Division of Neonatology, Children's Hospital of Fudan University, China
}

*Corresponding author: Ze Dong Jiang, Division of Neonatology, Children's Hospital of Fudan University, China.

Received Date: September 15, 2021

Published Date: September 28, 2021

\begin{abstract}
Very preterm infants who suffer from bronchopulmonary dysplasia (BPD), a major neonatal complication, often experience frequent episodes of hypoxaemia or prolonged hypoxaemia, resulting in significant chronic sublethal hypoxia (CSH). Whether the CSH occurring during the neonatal period exerts any adverse effect on the immature brainstem auditory system remains a topic of attracting considerable interest. With brainstem auditory evoked response (BAER) and particularly maximum length sequence (MLS) BAER, recent investigators have revealed that neonatal BPD damages functional integrity of the immature brainstem auditory system. There are significant abnormalities in the BAER components that reflect central neural function of the auditory brainstem. By comparison, there are only mild abnormalities in the BAER components that reflect peripheral neural function of the auditory brainstem. Clearly, CSH associated with neonatal BPD damage central neural conduction, suggesting impaired myelination and synaptic dysfunction, in the immature auditory brainstem, but exerts limited effect on peripheral neural function of the brainstem. These abnormalities tended to be improved after term date. CSH occurring during the neonatal period damages myelination of the central auditory system, which tends to be alleviated after term date.
\end{abstract}

\section{Introduction}

Considerable evidence indicates that neurodevelopmental disorders in infants born very prematurely often link to hypoxic events during the perinatal period. Oxygen deprivation or hypoxia is a major cause of neonatal brain impairment and subsequent long-term neurodevelopmental consequences. Acute and severe hypoxia which is often associated with ischaemia is well known to significantly damage the brainstem auditory system, leading to brainstem auditory lesions [1,2]. On the other hand, the understanding of whether chronic sublethal hypoxia (CSH) occurring during the neonatal period affects the brainstem auditory system remains rather limited. Not until recently, the studies of brainstem auditory evoked responses (BAERs) in very preterm infants who suffered from neonatal bronchopulmonary dysplasia (BPD) have significantly improved our understanding of the effect of CSH on the immature brainstem auditory system [3-10].

Hypoxia is particularly prevalent among those who are born very prematurely [11,12]. Many of the very preterm infants undergo chronic or prolonged periods of sublethal hypoxia due to various perinatal conditions or complications [13]. A typical and significant perinatal complication that is associated with CSH in very preterm infants is neonatal BPD [14]. Infants who suffer from BPD often experience frequent episodes of hypoxaemia or prolonged hypoxaemia. Such hypoxaemia plays an important role in the development of neurological impairment and deficit in infants with BPD. With the BAER, recent studies have revealed that these infants have major impairment in the central auditory system [3-10]. This article reviews recent advances in BAER studies in neonatal BPD. The abnormalities found in very preterm infants with BPD suggest poor myelination and synaptic dysfunction in the auditory brainstem, which may well be related to or due to CSH that occurs during the process of BPD. 


\section{Brainstem Auditory Impairment in Very Preterm Infants With CSH}

Previous experiments in animal models showed clear evidence that the immature cerebral cortex is adversely affected by CSH [15-17]. In human infants, CSH is also likely to affect the immature central auditory system. In very preterm infants, a typical and significant clinical complication or problem that is associated with CSH is neonatal BPD - a severe type of chronic lung disease. It is a major lung disease that causes hypoxaemia of pulmonary origin in infants who are born very prematurely $[14,18,19]$. The survivors of infants with BPD have a high incidence of neurological impairment and developmental deficits, such that BPD has increasingly become one of the highest risk factors of neurodevelopmental problems [18-26].

The BAER, also known as auditory brainstem response or ABR, is a non-invasive objective test, particularly suitable in very young or sick infants, and reflects functional integrity of the auditory brainstem [27]. The measurements of BAER variables are related to myelination and synaptic function along the brainstem auditory system of the developing brain. The BAER is very sensitive to arterial blood oxygen levels and hypoxia or hypoxia-ischaemia and is an important tool to assess cerebral and auditory function in infants after perinatal hypoxia or hypoxia-ischaemia $[27,28]$. A relatively new technique - the maximum length sequence (MLS) - has also been employed to record and process BAER $[27,29]$. The very highrate stimulation, known as stimulus "stress", in MLS could improve the detection of some neuropathology which may not be detected by presenting less stressful stimuli (i.e. low-rate stimulation) using conventional averaging techniques $[27,29]$.

Over the last decade, both conventional BAER and particularly MLS BAER have been used to study central auditory function in very preterm infants with BPD [3-10]. At term equivalent age, very preterm BPD infants were found to have major abnormalities in MLS BAER central components, that is, the components that mainly reflect or are related to central neural function, including wave $\mathrm{V}$ latency and I-V and particularly III-V interpeak intervals [4-7,9,10]. Such abnormalities were particularly significant at very high rates (455/sec and 910/sec). In contrast, no apparent abnormalities were found in the components that are mainly related to peripheral auditory function, including wave I and III latencies. There was also no major abnormality in the I-III interval, which reflects functional integrity of the more peripheral or caudal regions of the auditory brainstem, although this interval was slightly longer than that in the healthy very preterm infants at high rate-stimulation. These results imply that the increase in wave $\mathrm{V}$ latency and I-V interval in the infants with BPD was fundamentally produced by the significant increase in III-V interval that reflects functional integrity of the more central or rostral regions of the auditory brainstem. Similar abnormalities were seen in the BPD infants when their BPD was diagnosed shortly before term date [8].
Clearly, in very preterm infants with BPD, neural conduction mainly related to myelination in the more central or rostral regions of the auditory brainstem, is delayed or impaired during the course of BPD. The slopes of wave V latency-rate function, and I-V and particularly III-V interval-rate functions tended to be increased in the infants with BPD. This increased stimulus ratedependent change in MLS BAER central components suggests that auditory neurons in the more central regions of the auditory brainstem in infants with BPD are vulnerable to physiological/ temporal challenge of acoustic stimulation, resulting in a decreased efficacy of central synaptic transmission. Therefore, in addition to myelination, central synaptic efficacy in the auditory brainstem is also affected by CSH during the course of BPD.

On the other hand, the BPD infants did not show any apparent abnormalities in MLS BAER components that are mainly related to peripheral auditory function, including wave I and III latencies. There was also no appreciable abnormality in the I-III interval, which reflects the functional status of more peripheral or caudal regions of the auditory brainstem. These results suggest that functional status of the more peripheral regions of the auditory brainstem in BPD infants is relatively intact or only slightly affected. Therefore, CSH does not have a major effect on functional integrity of the more peripheral regions of the auditory brainstem.

The aforementioned major abnormalities in the MLS BAER variables that mainly reflect central neural function suggest a significant delay in neural conduction, mainly reflecting impaired myelination, in the more central regions of the auditory brainstem [4-10]. The increased click rate-dependent change in MLS BAER central components mainly reflects a decreased efficacy of synaptic transmission. Therefore, $\mathrm{CSH}$ associated with BPD has a detrimental effect on functional integrity of the auditory brainstem in very preterm infants with BPD. However, peripheral neural function of the auditory system does not seem to be significantly affected. By comparison, the amplitudes of BAER waves I, III and V in the infants with BPD were generally similar to those in the healthy very preterm infants at all click rates. The $\mathrm{V} / \mathrm{I}$ and $\mathrm{V} / \mathrm{III}$ amplitude ratios did not show any significant differences from those in the healthy very preterm infants. Such relatively normal wave amplitudes are in sharp contrast to the abnormal findings in wave latencies and intervals. Neonatal BPD does not seem to exert any major effect on neural origin of the amplitudes, which is more related to the neuronal function of MLS BAER components, although it has a significant effect on the neural origin of wave latencies and intervals, which are more related to neural conduction and myelination of the brainstem.

\section{Possible Mechanisms Underlying the Brainstem Auditory Impairment}

The pathogenesis of neonatal BPD is complex and multifactorial, including very immaturity, respiratory distress syndrome, patent ductus arteriosus, disrupted alveolar and capillary development, 
pulmonary interstitial emphysema, oxygen toxicity, perinatal infection and inflammation or genetic susceptibility. Over the last two decades, there has been no significant change in the incidence of BPD, but the pathophysiology has changed with the substantial improvement in the management in very and extremely preterm infants [31,32]. Infants with BPD have frequent episodes of hypoxaemia or prolonged hypoxaemia, leading to CSH [14]. The auditory impairment in BPD could be related to various risk factors and causes, particularly CSH as a result of the frequent episodes of hypoxaemia or prolonged hypoxaemia during the course of BPD. The CSH may play a significantly adverse role in the impairment. Since none of our BPD infants had any concomitant major brain lesions that may exert confounding effects on the MLS BAER, the major MLS BAER abnormalities found in these BPD infants are most likely to be mainly attributed to the frequent episodes of hypoxaemia or prolonged sublethal hypoxaemia that is associated with BPD [10].

Hypoxaemia disturbs the metabolism of neurons, depresses the electrophysiological function of synapses, and interferes with nerve conduction, leading to neural impairment and dysfunction of the brain. Hypoxaemia occurring during early life often results in neurodevelopmental disorders, including auditory impairment [28,33-39]. In neonatal BPD, the prolonged hypoxaemia is often associated with periods of oxygen desaturation, as well as suboptimal respiratory mechanics [14]. Infants with BPD are invariably associated with therapy in high oxygen concentrations for prolonged periods. The prolonged exposure to high oxygen concentrations has complex biochemical microscopic and gross anatomical effects on lung tissues, which can injure the immature lung and cause further hypoxaemia.

In addition, infants with neonatal BPD are often associated with other perinatal conditions or complications, which may also exert certain adverse effects on the immature auditory system. However, $\mathrm{CSH}$ remains the major adverse effect that contributes to the BAER abnormalities seen in the very preterm infants with BPD. The central auditory system experiences considerable synaptogenesis during early life, and there is a tremendous growth of dendrites after term date. Myelination occurs during the second growth spurt of the brain, which takes place in the second half of gestation and lasts well into the second postnatal year of later. Neonatal BPD in very preterm infants occurs before term, corresponding to later phase of the second half of gestation. The rapid myelination of the auditory brainstem occurring during this period can be interfered by $\mathrm{CSH}$ associated with neonatal BPD. Neural conduction in the developing auditory brainstem is closely related to myelination in the auditory system. The brainstem auditory system, including relay nuclei and fibres, develops myelination in the foetal and early postnatal periods. The significant increase in the I-V and, in particular, III-V intervals in our very preterm infants with BPD indicates impaired neural conduction and so impaired myelination in the more central regions of the auditory brainstem.

\section{Alleviation of Brainstem Auditory Impairment After Term}

To explore any changes after term date in the auditory impairment detected at term in very preterm infants enduring $\mathrm{CSH}$, we recorded and analysed BAER in a group of BPD infants at 3 months of postconceptional age [3]. The latencies of BAER waves $\mathrm{I}$ and $\mathrm{V}$ in the BPD infants were similar to those in the normal controls. Wave III latency was slightly shorter in the cases than the controls. The I-V interval was similar in the two groups of infants. However, the I-III interval in the BPD infants was shorter than in the controls, whereas the III-V interval was longer than in the controls. The amplitudes of BAER waves I, III and V in the BPD infants all tended to be smaller than in the controls, but none of the amplitudes differed significantly between the two groups. No significant differences were found in the V/I and V/III amplitude ratios between the two groups. Thus, at 3 months of age these BPD infants did not show any obvious abnormalities in the BAER wave III and $\mathrm{V}$ latencies and the I-V interval, but there was a marginal shortening in the I-III interval and a marginal increase in the III-V interval.

The marginal shortening in the I-III interval in the BPD infants suggests a possible acceleration in myelination and maturation of the peripheral regions of the brainstem auditory system after term. These BPD infants were all born at 29 weeks or less of gestation. The much earlier exposure to the extrauterine sound environment and the associated excessive exogenous noise in the intensive care unit could accelerate myelination and maturation of the auditory system, leading to a relative shortening of the I-III interval $[29,30,40]$. The marginal increase in the III-V interval in the BPD infants, which was much less significant than the increase seen at term, suggests minor auditory impairment at central or rostral regions of the brainstem. These mild BAER abnormalities suggest minor impairment in both the peripheral and central auditory systems at 3 months of postconceptional age. Obviously, the major auditory impairment detected at term in the BPD infants was alleviated after term. The marginal shortening in the I-III interval and the marginal increase in the III-V interval suggest possibly accelerated maturation in the more peripheral part of the auditory brainstem and delayed maturation in the more central part. Whether these minor abnormalities will further change with increasing age is a subject of further study.

\section{Conclusion}

Recent conventional BAER and particularly MLS BAER studies in very preterm infants with BPD have discovered major abnormalities that are significantly related to CSH during the neonatal period. The major abnormality is a significant increase or prolongation in the BAER components that mainly reflect central auditory function. This implies a significant delay in neural conduction, mainly reflecting impaired myelination in the more central regions of the auditory brainstem. On the other hand, no major abnormality was 
found in the peripheral neural function of the auditory brainstem. Clearly, CSH associated with neonatal BPD significantly damages myelination and synaptic function in the immature central auditory system, but does not significantly damage neural function in the peripheral auditory system. The impaired myelination and synaptic function tends to be alleviated after term date. These findings shed light on our understanding of the effect of neonatal CSH on functional integrity of the very immature auditory system and may have important clinical implications with regard to studying therapeutic measures to improve brainstem auditory outcome in infants with CSH.

\section{Acknowledgement}

None.

\section{Conflict of Interest}

No conflict of interest.

\section{References}

1. Natsume J, Watanabe K, Kuno K, Hayakawa F, Hashizume Y (1995) Clinical, neurophysiologic, and neuropathological features of an infant with brain damage of total asphyxia type (Myers). Pediatr Neurol 13: 61-64.

2. Pasternak JF (1993) Hypoxic-ischemic brain damage in the term infants: lessons from the laboratory. Pediatr Clin North Am 40:1061-1072.

3. Jiang ZD (2017) Auditory impairment is alleviated after term in infants with neonatal chronic lung disease. Acta Paediatr 106: 926-9.

4. Jiang ZD, Brosi DM, Wilkinson AR (2006) Brainstem auditory function in very preterm infants with chronic lung disease: delayed neural conduction. Clin Neurophysiol 117: 1551-1559.

5. Jiang ZD, Yin R, Wilkinson AR (2007) Brainstem auditory evoked responses in very low birthweight infants with chronic lung disease. Eur J Pediatr Neurol 11: 153-159.

6. Jiang ZD, Brosi DM, Chen C, Wilkinson AR (2009) Brainstem response amplitudes in neonatal chronic lung disease and differences from perinatal asphyxia. Clin Neurophysiol 120: 967-973.

7. Jiang ZD, Brosi DM, Wilkinson AR (2010) Differences in impaired brainstem conduction between neonatal chronic lung disease and perinatal asphyxia. Clin Neurophysiol 121: 725-733

8. Jiang ZD, Wang C (2016) Abnormal findings in brainstem auditory evoked response at 36-37 weeks of age in babies with neonatal chronic lung disease. Early Hum Dev 103: 161-165.

9. Wang C, Jiang ZD (2018) Brainstem auditory abnormality in extremely premature babies and the impact of neonatal bronchopulmonary dysplasia. Acta Obstet Gynecol Scand 97: 545-551

10. Wilkinson AR, Brosi DM, Jiang ZD (2007) Functional impairment of the brainstem in infants with bronchopulmonary dysplasia. Pediatrics 120 362-371.

11. Hack M, Wright LL, Shankaran S, Tyson JE, Horbar JD, et al. (1995) Verylow-birth-weight outcomes of the National Institute of Child Health and Human Development Neonatal Network, November 1989 to October 1990. Am J Obstet Gynecol 172: 457-464.

12. Vohr BR, Msall ME (1997) Neuropsychological and functional outcomes of very low birth weight infants, Semin Perinatol 21: 202-220.

13. Poets CF, Stebbens VA, Richard D, Southall DP (1995) Prolonged episodes of hypoxia in preterm infants indetectable by cardiorespiratory monitors. Pediatrics 95: 860-863.

14. Greenough A, Milner AD (2005) Pulmonary disease of the newborn: Chronic lung disease. In: Rennie JM, editor. Roberton's Textbook of Neonatology, 4th ed. Edinburgh, Schotland: Elsevier Churchill Livingstone: 554-572.
15. Curristin SM, Cao A, Stewart WB, Zhang H, Madri JA, et al. (2002) Disrupted synaptic development in the hypoxic newborn brain. Proc Natl Acad Sci USA 99: 15729-15734.

16. Schwartz MLS, Vaccarino F, Chacon M, Yan WL, Ment LR, et al. (2004) Chronic neonatal hypoxia leads to long term decreases in the volume and cell number of the rat cerebral cortex. Semin Perinatol 28: 379-388.

17. Weiss J, Takizawa B, McGee A, Stewart WB, Zhang H, et al. (2004) Neonatal hypoxia suppresses oligodendrocyte Nogo-A and increases axonal sprouting in a rodent model for human prematurity. Exp Neurol 189: 141-149.

18. Katz-Salamon M, Gerner EM, Jonsson B, Lagercrantz H (2000) Early motor and mental development in very preterm infants with chronic lung disease. Arch Dis Child 83: F1-6.

19. Murphy BP, Inder TE, Huppi PS, Warfield S, Zientara GP, et al. (2001) Impaired cerebral cortical gray matter growth after treatment with dexamethasone for neonatal chronic lung disease. Pediatrics 107: 217-221.

20. Böhm B, Katz-Salamon M (2003) Cognitive development at 5.5 years of children with chronic lung disease of prematurity. Arch Dis Child Fetal Neonatal Ed 88: 101-105.

21. van Katwyk S, Augustine S, Thébaud B, Thavorn K (2020) Lifetime patient outcomes and healthcare utilization for Bronchopulmonary dysplasia (BPD) and extreme preterm infants: a microsimulation study. BMC Pediatr 20: 136

22. Perlman JM (2001) Neurobehavioral deficits in premature graduates of intensive care - potential medical and neonatal environmental risk factors. Pediatrics 108: 1339-1348.

23. Short EJ, Klein NK, Lewis BA, Fulton S, Eisengart S, et al. (2003) Cognitive and academic consequences of bronchopulmonary dysplasia and very low birth weight: 8-year-old outcomes. Pediatrics 112: e359.

24. Thompson DK, Warfield SK, Carlin JB, Pavlovic M, Wang HX, et al. (2007) Perinatal risk factors altering regional brain structure in the preterm infant. Brain 130: 667-677

25. Tracy MK, Berkelhamer SK (2019) Bronchopulmonary Dysplasia and Pulmonary Outcomes of Prematurity. Pediatr Ann 48: e148-e153.

26. Vohr BR, Wright LL, Dusick AM, Mele L, Verter J, et al. (2000) Neurodevelopmental and functional outcomes of extremely low birth weight infants in the National Institute of Child Health and Human Development Neonatal Research Network, 1993-1994. Pediatrics 105: 1216-1226.

27. Wilkinson AR, Jiang ZD (2006) Brainstem auditory evoked response in neonatal neurology. Semin Fet Neonatol Med 11: 444-451.

28. Volpe JJ (2001) Perinatal brain injury. From pathogenesis to neuroprotection. Ment Retard Dev Disabil Res Rev 7: 56-64.

29. Jiang ZD (2015) Evoked potentials in pediatric brainstem lesions. In: G Galloway, editor. Clinical Neurophysiology in Pediatrics: A Practical Approach to Neurodiagnostic Testing and Management. New York: Demos Medical Publishing, LLC P. 187-213.

30. Wilkinson AR, Jiang ZD (2006) Brainstem auditory evoked response in neonatal neurology. Semin Fet Neonatol Med 11: 444-451.

31. Hwang JS, Rehan VK (2018) Recent Advances in Bronchopulmonary Dysplasia: Pathophysiology, Prevention, and Treatment. Lung 196: 129-138.

32. Kalikkot Thekkeveedu R, Guaman MC, Shivanna B (2017) Bronchopulmonary dysplasia: A review of pathogenesis and pathophysiology. Respir Med 132: 170-177.

33. Gonzalez FF, Miller SP (2006) Does perinatal asphyxia impair cognitive function without cerebral palsy? Arch Dis Child Fetal Neonatal Ed 91: F454-459.

34. Jiang ZD (2008) Brainstem electrophysiological changes after perinatal hypoxia ischemia. In: Hämäläinen E, editor. New Trends in Brain Hypoxia Ischemia Research. New York: Nova Science Publishers: 203-220.

35. Johnston M, Trescher WH, Ishida A, Nakajima W (2001) Neurobiology of hypoxic-ischemic injury in the developing brain. Pediatr Res 49: 735741 
36. Levene MI (2001) The newborn infant. In: Levene MI, Chervenak FA, Whittle M, editors. Fetal and neonatal neurology and neurosurgery, 4th ed. Edinburgh: Churchill-Livingstone: 471-504.

37. Levene MI, Evans DJ (2005) Neurological problems in the newborn: Hypoxic-ischaemic brain injury. In: Rennie JM, editor. Roberton's Textbook of Neonatology, 4th ed. Edinburgh: Elsevier Churchill Livingstone: 11281148.

38. Marlow N, Rose AS, Rands CE, Draper ES (2005) Neuropsychological and educational problems at school age associated with neonatal encephalopathy. Arch Dis Child 90: 380-387.
39. Rennie JM, Hagmann CF, Robertson NJ (2007) Outcome after intrapartum hypoxic ischaemia at term. Semin Fetal Neonatal Med 12: 398-407.

40. Jiang ZD, Brosi D, Wu YY, Wilkinson AR (2009) Relative maturation of the peripheral and central regions of the auditory brainstem from preterm to term and the influence of preterm birth. Pediatr Res 65: 657-662. 